\title{
ArcheoSciences
}

Revue d'archéométrie

$36 \mid 2012$

Varia

\section{Du cadastre ancien au graphe. Les dynamiques spatiales dans les sources fiscales médiévales et modernes}

From ancient cadastre to graph model. Spatial dynamics in medieval and modern tax sources

Mélanie Le Couédic, Samuel Leturcq, Xavier Rodier, Florent Hautefeuille, Etienne Fieux et Bertrand Jouve

\section{OpenEdition \\ Journals}

Édition électronique

URL : https://journals.openedition.org/archeosciences/3758

DOI : 10.4000/archeosciences.3758

ISBN : 978-2-7535-2243-5

ISSN : 2104-3728

Éditeur

Presses universitaires de Rennes

\section{Édition imprimée}

Date de publication : 31 décembre 2012

Pagination : 71-84

ISBN : 978-2-7535-2241-1

ISSN : 1960-1360

Référence électronique

Mélanie Le Couédic, Samuel Leturcq, Xavier Rodier, Florent Hautefeuille, Etienne Fieux et Bertrand Jouve, "Du cadastre ancien au graphe. Les dynamiques spatiales dans les sources fiscales médiévales et modernes », ArcheoSciences [En ligne], 36 | 2012, mis en ligne le 31 décembre 2014 consulté le 01 février 2022. URL : http://journals.openedition.org/archeosciences/3758; DOI : https:// doi.org/10.4000/archeosciences.3758 


\title{
Du cadastre ancien au graphe Les dynamiques spatiales dans les sources fiscales médiévales et modernes
}

\author{
From Ancient Cadastre to Graph Model \\ Spatial Dynamics in Medieval and Modern Tax Sources
}

\author{
Mélanie Le Couédic*, Samuel LeturcQ*, Xavier Rodier*, \\ Florent Hautefeuille**, Etienne Fieux ${ }^{* * *}$ et Bertrand Jouve****
}

\begin{abstract}
Résumé : Les documents fiscaux médiévaux et modernes (terriers, compoix, cadastres...) offrent une information spatiale riche. Des territoires entiers sont décrits parcelle par parcelle à des époques successives. Les historiens ne parviennent toutefois jamais à corréler ces différents états de l'information spatiale sur la longue durée, d'autant qu'on ne dispose d'aucun plan parcellaire avant les XVII ${ }^{\mathrm{e}}$-XVIII ${ }^{\mathrm{e}}$ siècles. C'est ce verrou méthodologique qu'il s'agit de dépasser en proposant, dans le cadre de l'ANR Modelespace, une modélisation des parcellaires anciens décrits dans les documents fiscaux en mettant en œuvre les propriétés topologiques de la théorie des graphes. La conversion des données spatiales en graphes doit permettre de mettre en place un langage commun entre les documents historiques. L'article traite des choix méthodologiques fondamentaux et de la démarche utilisée pour transformer l'information spatiale des plans anciens en graphes susceptibles d'être appariés non seulement entre eux, mais encore avec des graphes issus de matrices cadastrales sans plan. Au final, c'est au sein d'un Système d'Information Géographique que devra être testée la pertinence des possibilités de comparaisons en étudiant les recompositions parcellaires selon des opérateurs spatio-temporels de changement (création, disparition, stabilité, dilatation, contraction, fusion, fission, déformation).
\end{abstract}

\begin{abstract}
Medieval and modern tax documents (fieldbooks, "compoix", cadasters...) give a rich spatial information. Whole territories are described plot per plot at different succesive periods. Nevertheless, historians don't know how to relate different states of spatial information on the long term. Moreover, there are no plot plans before the $17^{\text {th }}$ and $18^{\text {th }}$ century. We want to overcome this methodological lock with the following proposition : modelling ancient plots described in tax documents using the topological proprieties of the graph theory. The translation of the spatial data into graphs should allow to set up a commun language between the historical documents, mapped and not mapped. The article talks about the fondamental method choices and the used process to transform the spatial information of ancient plans into graphs which will allow us not only to compare them together, but also to compare them with cadastral registers that have no plan. Finally, it is within a Geographic Information System that will be tested the pertinence of comparing possibilities while studying the plot changes according to the spatiotemporal operators of changement (creation, disappearance, stability, dilatation, contraction, fusion, fission, deformation).
\end{abstract}

Mots clé : Terrier, cadastre, Systèmes d'Information Géographique (SIG), Théorie des graphes, Moyen Âge, période moderne.

Keywords: Fieldbook, cadaster, Geographic Information Systems (GIS), Graph theory, Medieval Ages, Modern period.

* UMR 7324, Laboratoire Archéologie et Territoires, CITERES, Université François-Rabelais de Tours, CNRS, BP 60449, 37204 Tours cedex 03 (France).(melanie.lecouedic@gmail.com) (samuel.leturcq@univ-tours.fr) (xavier.rodier@univ-tours.fr)

** UMR 5136, Laboratoire FRAMESPA, Université Toulouse II Le Mirail- 5, allée Antonio Machado, 31058 Toulouse cedex 09 (France) (florent. hautefeuille@univ-tlse2.fr)

*** UMR 5219, Institut mathématique de Toulouse, Université Toulouse III Paul Sabatier-118 route de Narbonne, 31062 Toulouse cedex 09 (France). (fieux@math.univ-toulouse.fr)

**** EA 3083, Laboratoire ERIC, Université de Lyon, Lumière Lyon 2 - 5, av. Pierre Mendès-France, 69600 Bron (France). (bertrand.jouve@univlyon2.fr)

rec. jul. 2011 ; acc. mar. 2012

ArCHeoSCIEnCes, revue d'archéométrie, 36, 2012, p. 71-84 


\section{INTRODUCTION}

Les recherches sur l'histoire récente des sociétés, sur l'histoire du paysage ou du climat reposent toutes sur des données rattachées à un référentiel cartographique précis. C'est à partir du XIX ${ }^{\mathrm{e}}$ siècle, voire dès le XviII ${ }^{\mathrm{e}}$ siècle dans certaines régions pionnières d'Europe, que se systématise la cartographie de l'espace à des échelles précises (généralement le $1 / 2500^{\circ}$ ) dans un objectif fiscal. Avant cette cadastration systématique des territoires, la rareté et l'hétérogénéité de la documentation cartographique constituent des freins considérables à l'appréhension des phénomènes spatiaux anciens. En effet, le chercheur est confronté, selon le territoire qu'il étudie, soit à la rareté (voire l'absence complète) des données localisables, soit à leur extraordinaire profusion. Dans ce second cas de figure, cette richesse est aussi préjudiciable puisqu'elle rend impossible l'utilisation de ces données en l'absence d'un instrument de traitement informatique.

Dans la masse documentaire offerte aux historiens et aux archéologues pour reconstituer les dynamiques paysagères médiévales et modernes (XIII ${ }^{\mathrm{e}}$-XVIII ${ }^{\mathrm{e}}$ siècle), il est une catégorie de sources particulièrement riches en données spatialisables : les terriers et les compoix. Nous disposons, pour la France seule, de plusieurs dizaines de milliers de registres fiscaux antérieurs au XIX $X^{e}$ siècle, dont les plus anciens remontent au XIII ${ }^{\mathrm{e}}$ siècle. Les terriers correspondent à des sources fiscales privées tandis que les compoix sont des sources fiscales publiques présentes au sud de l'Europe. Ces documents, créés dans le but de servir à des prélèvements assis sur la possession foncière, généralement conservés sous la forme de registres, parfois accompagnés de plans pour les documents les plus récents, peuvent être considérés comme les ancêtres du cadastre actuel. Présent aussi bien en France que dans le reste de l'Europe, ce type de source livre une information abondante et de grande qualité, dont l'intérêt majeur réside dans la forme de l'enregistrement, à savoir des listes de noms et de terres, avec une multitude de précisions, et notamment à la fin le montant de la redevance à payer (l'allivrement lorsqu'il s'agit d'un cadastre ou d'un compoix). La standardisation de l'enregistrement de ces longues séries (fréquemment plusieurs milliers d'items) rend particulièrement propice l'utilisation d'outils d'analyse, en particulier statistiques. Mais surtout, dans la mesure où les déclarations consignent pour chaque parcelle de terre non seulement le nom et la profession du propriétaire, parfois le nom de l'exploitant, mais surtout très souvent la superficie, le mode d'utilisation de la terre (terre labourable, vigne, pré, pâquis, bois, lotissement...), une localisation par un microtoponyme, les noms des propriétaires des parcelles adjacentes (confronts)..., il est possible de replacer les parcelles les unes par rapport aux autres au sein d'un territoire, en s'aidant du plan-terrier s'il existe, sinon du cadastre napoléonien. Sont envisageables alors diverses questions historiques portant sur la structuration des territoires (structures paysagères, répartition de l'utilisation des terres, organisation de la propriété et de l'exploitation agricole...).

Bien que ces documents fiscaux médiévaux et modernes offrent une information spatiale très riche avec des territoires entiers précisément décrits parcelle par parcelle et à des époques successives, les historiens ne parviennent toutefois jamais à corréler ces informations spatiales sur la longue durée, ce qui permettrait de préciser les dynamiques de recomposition à l'œuvre. Cette difficulté est renforcée par l'absence de plan parcellaire avant les XVII ${ }^{\mathrm{e}}$-XVIII ${ }^{\mathrm{e}}$ siècles. Le chercheur est ainsi obligé de se lancer dans la reconstitution d'un puzzle en utilisant les précisions topographiques, microtoponymiques, et surtout les mentions de confronts. Ce travail, long et fastidieux, est difficile car il existe des lacunes dans les informations (par exemple certains petits confronts ne sont pas décrits) et des ambiguïtés. Il est cependant envisageable pour reconstituer le parcellaire d'une zone restreinte, mais en aucun cas pour l'ensemble d'un terroir. Les historiens exploitent, de fait, essentiellement la documentation récente à partir du moment où ils disposent de plans. L'absence d'un référent cartographique complique l'usage et le traitement de la masse d'informations et, a fortiori, les possibilités d'observation des transformations du parcellaire d'un état à l'autre afin d'améliorer la compréhension des dynamiques spatiales. Le traitement de l'information spatiale des terriers et compoix est ainsi restée le plus souvent limitée à des initiatives ponctuelles, développant des solutions " artisanales ", dans lesquelles le caractère dynamique était peu présent (Claveirole et Pélaquier 2001; Brunel et al., 2002). Le colloque de Rome de 1985 (Biget et al., 1989) constitue sur cette thématique un jalon pionnier et essentiel de la recherche. Les constats que nous faisons aujourd'hui y sont déjà présents, mettant l'accent sur la nécessité de créer un outil capable de gérer la masse des données spatiales présentes dans les cadastres urbains médiévaux. Un article envisage même, dès cette époque, la possibilité de l'utilisation d'un objet mathématique, les graphes, pour accélérer le processus de reconstitution des parcellaires urbains médiévaux à partir des compoix (Montpied et Rouault, 1989). Ce projet était déjà exprimé dans un court article de la revue Le médiéviste et l'ordinateur (Montpied et Rouault, 1982). L'expérience proposée s'est alors essentiellement limitée à la construction du graphe (les parcelles sont réduites à un point et deux parcelles voisines correspondent à deux sommets voisins dans le graphe), puis aux repérages simplement visuels «d'accidents topolo- 
giques » sur le graphe. À notre connaissance, aucune avancée concernant la mise en place de méthodes de ce type n'a été proposée pour la " reconstruction » de parcellaires anciens depuis ces travaux du milieu des années 1980, bien que les recherches les plus récentes soulignent cette carence technique (Leturcq, 2007; Truel, 2007; Conesa, 2007; Poirier, 2010). Des éléments de théorie des graphes sont de plus en plus utilisés en écologie des paysages (par exemple : Dale et Fortin, 2010; Cantwell et Forman, 1993; Urban et Keitt, 2001) fournissant un cadre mathématique pour la création de modèles d'écosystèmes. Bien que le territoire y soit aussi représenté par un graphe d'adjacence et s'il est important de suivre la façon dont cette communauté scientifique s'approprie et développe cet outil mathématique, leurs questions concernent plutôt la création d'indicateurs pour évaluer la structure des écosystèmes.

Reprenant cette idée d'utiliser le concept de graphe mathématique (Berge, 1958), nous présentons dans ce papier quelques avancées méthodologiques afin de permettre l'exploitation des informations spatiales contenues dans des grandes séries de documents fiscaux non cartographiés. Ce projet est mené dans le cadre du programme ANR MODELESPACE ${ }^{1}$. Plus précisément, les objectifs de ce programme sont d'aller au-delà des reconstructions manuelles des plans parcellaires en générant un outil qui permette de mener, à terme, d'ambitieux projets d'analyse des dynamiques spatiales de territoires urbains ou ruraux pour lesquels on dispose de plusieurs terriers, compoix et cadastres qui sont autant d'enregistrements successifs de l'organisation du territoire. C'est donc à un changement d'échelle qu'invite cet outil de modélisation des dynamiques spatiales, puisqu'il doit permettre d'intégrer dans une même base de données les états successifs d'un même espace, d'offrir une modélisation graphique de chacun de ces états, et d'étudier les dynamiques spatiales par comparaison de ces différentes représentations. L’objectif n'est donc pas de créer un outil de reconstitution automatique d'un parcellaire au plus proche de la réalité passée; ce serait un objectif non seulement peu réaliste, mais encore fondé sur une conception statique des territoires et des paysages.

Les travaux des mathématiciens en théorie des graphes ont été nombreux ces 25 dernières années, notamment dans le cadre de leur application aux sciences sociales. L'utilisation de la théorie des graphes pour l'analyse des grands réseaux d'interactions (réseaux sociaux, réseau de l'internet, réseaux d'interactions de protéines, réseaux de transports...) a forte-

1. ANR-09-BLAN-0322-02 « Modélisation des espaces préindustiels». Ce projet 2010-2012 est dirigé par Florent Hautefeuille (FRAMESPA), Bertrand Jouve (IMT) et Samuel Leturcq (Citeres-LAT). Le site internet est consultable à cette adresse : http://w3.modele-espace.univ-tlse2.fr/ ment progressé dans les années 2000 avec la possibilité d'accéder directement en ligne à de grandes masses de données et le développement de nouveaux modèles mathématiques de graphes (Watts et Strogatz, 1998; Barabási et Albert, 1999). Nous proposons de coupler les outils maintenant classiques de l'analyse des réseaux avec des outils de topologie ${ }^{2}$ pour étudier des graphes où chaque noud représente une parcelle et deux nœuds sont liés par une arête si les deux parcelles sont voisines géographiquement. Depuis l'article précurseur de Atkin (Atkin 1977), des travaux récents (Babson et al., 2006; Chen et al., 2001; Dochterman, 2009; Boulet et al., 2010) ont permis de progresser dans les interactions entre graphe et topologie. Cette étude s'inscrit dans l'application de cette dynamique à une base de données réelle.

\section{MÉTHOde}

\section{Démarche générale}

La démarche consiste à transformer l'information spatiale, issue d'une part des registres, d'autre part des plans parcellaires anciens, en graphes (appelés " graphes duals") dans lesquels chaque parcelle correspond à un sommet et chaque relation de voisinage (adjacence visible sur un plan ou décrite dans les documents par la mention des confronts) correspond à une arête. Suivant le type de source, cartographiée ou non, le travail est mené selon deux processus différents (figure 1).

Le premier processus consiste en la construction d'un outil de saisie et d'analyse des données spatiales des documents fiscaux permettant la construction de graphes. La base de données est développée de manière à pouvoir intégrer dans un même ensemble les données de plusieurs registres et matrices en deux étapes. Dans la première étape, il s'agit de saisir l'ensemble des informations contenues dans les registres fiscaux, en tenant compte de la variété des formes rédactionnelles de ces registres, tout en proposant des outils permettant d'associer des termes désignant la même personne ou le même lieu apparaissant sous des orthographes différentes. La seconde étape propose des algorithmes d'appariement de parcelles par leurs liens de voisinage, ce qui permet le basculement

2. La topologie, ou science des voisinages, est une branche des mathématiques créée par le mathématicien français Poincaré à la fin du XIX ${ }^{e} s$. pour pouvoir formaliser des raisonnements de nature qualitative. La topologie s’intéresse aux relations de voisinage entre les objets localisés géographiquement ou non. Elle permet de formaliser mathématiquement une approche, une description qualitative. Les relations de voisinage entre les objets s'expriment en termes de continuité, de contiguïté, de proximité, d'adjacence, de connectivité. Une modification des relations spatiales entraîne une modification de la topologie (Pirot, 2004 : 426). 


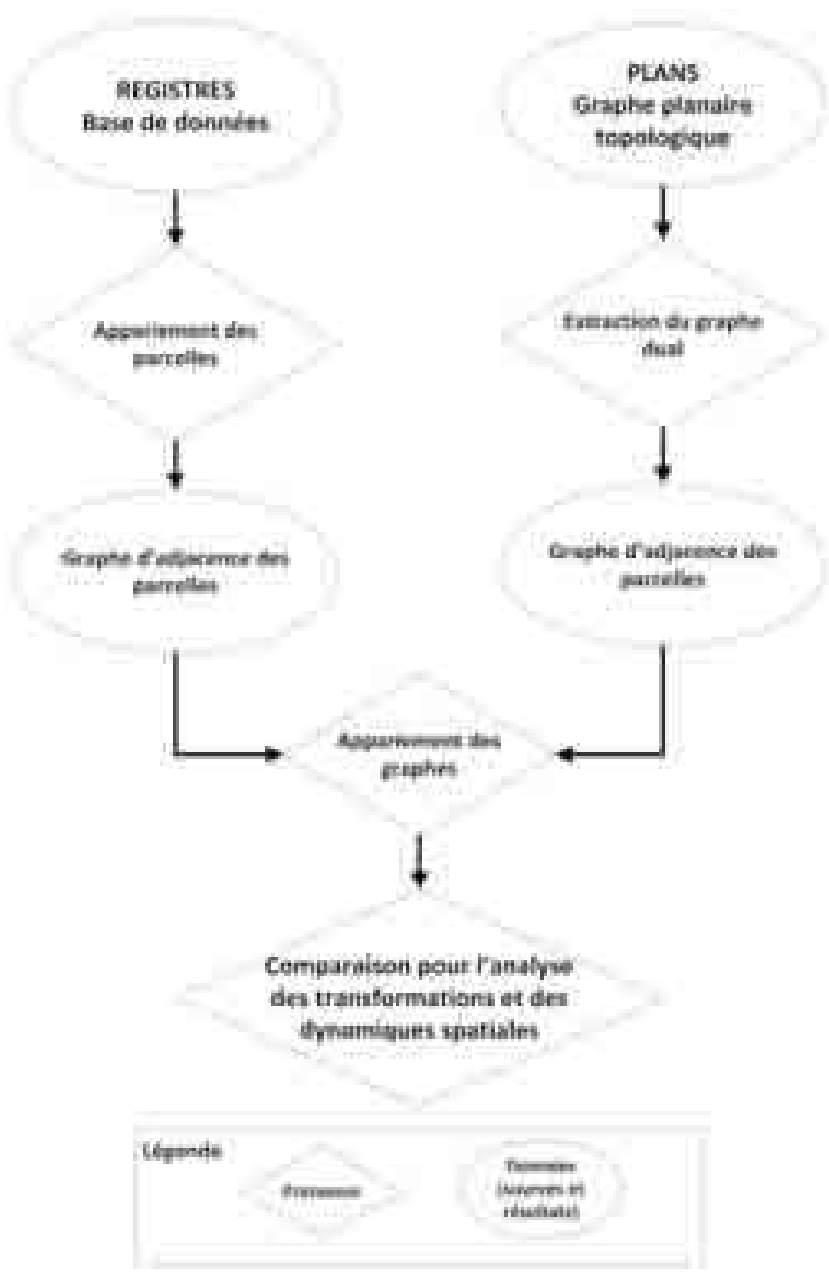

Figure 1 : Étapes de traitement des registres et des plans. Figure 1: Stages of processing of registers and plans.

semi-automatique des données issues des registres vers des graphes d'adjacence de parcelles. Les informations contenues dans les registres sont ainsi dépouillées et rentrées dans une base de données.

Le second processus, sur lequel cet article porte en priorité, consiste à extraire le graphe dual (Berge 1958 : 206-217) des plans parcellaires anciens dans un Système d'Information Géographique (SIG).

\section{La construction du graphe dual à partir du plan parcellaire}

À partir des plans originaux, il s'agit d'abord de vectoriser le plan selon une structure topologique de manière à obtenir le graphe planaire topologique du parcellaire ${ }^{3}$. Les sommets de ce graphe sont les points d'intersection des frontières des parcelles et les arcs sont les frontières des parcelles. De ce graphe, on extrait le graphe dual dans lequel chaque parcelle est représentée par un sommet et deux parcelles voisines sont reliées par un arc. Dans ce graphe dual, on affecte les attributs des parcelles aux sommets qui les représentent afin d'obtenir ce que nous appelons le graphe d'adjacence des parcelles. Une illustration de ces différents graphes est donnée en figure 2 .

Les étapes qui ont été empruntées pour la construction du graphe d'adjacence du plan parcellaire ancien et du plan terrier sont les suivantes :

-n umérisation,

- vectorisation des plans avec un logiciel de DAO (Adobe Illustrator),

- import des plans dans un logiciel SIG (ArcGis),

-g éoréférencementd esp lans,

- liaison du plan et de l'état de section pour lier les informations géométriques aux données attributaires,

- prise en compte de la relation entre les chemins et les parcelles (cf. infra),

- vérification et correction de la topologie pour l'obtention d'un graphe planaire topologique du parcellaire,

- extraction du graphe d'adjacence (cf. infra).

Dans l'idéal, dans le cas où les plans seraient directement intégrés et saisis dans le Système d'Information Géographique, on peut résumer les étapes empruntées pour le passage des plans originaux au graphe dual du parcellaire selon le schéma en figure 2.

Par ailleurs, la construction du graphe dual à partir du graphe planaire du parcellaire nécessite plusieurs choix et conditions préalables, dès la vectorisation du plan. D'abord il est important que le plan obtenu forme un pavage complet de l'espace, sans aucun trou ni aucune superposition entre les parcelles; il s'agit d'une question de topologie. Lors du dessin de deux parcelles voisines, les deux polygones doivent avoir une arête commune. Il s'agit donc de vérifier la topologie du tracé, souvent source d'erreur. Si cette étape est souvent longue et fastidieuse, les outils de topologie a priori, lors du dessin, ou a posteriori, en correction, offerts par le logiciel ArcInfo permettent une vérification efficace et relativement rapide. Ensuite, la vectorisation du plan demande de

3. Un plan parcellaire est un graphe planaire topologique. En effet « Un graphe $\mathrm{G}$ est planaire s'il est possible de le représenter sur un plan de sorte que les sommets soient des points distincts et les arcs des courbes simples ne se rencontrant pas en dehors de leurs extrémités. Un graphe planaire topologique est une représentation d'un graphe planaire G sur un plan " (Pirot2004 : 422-423; Berge 1970). 


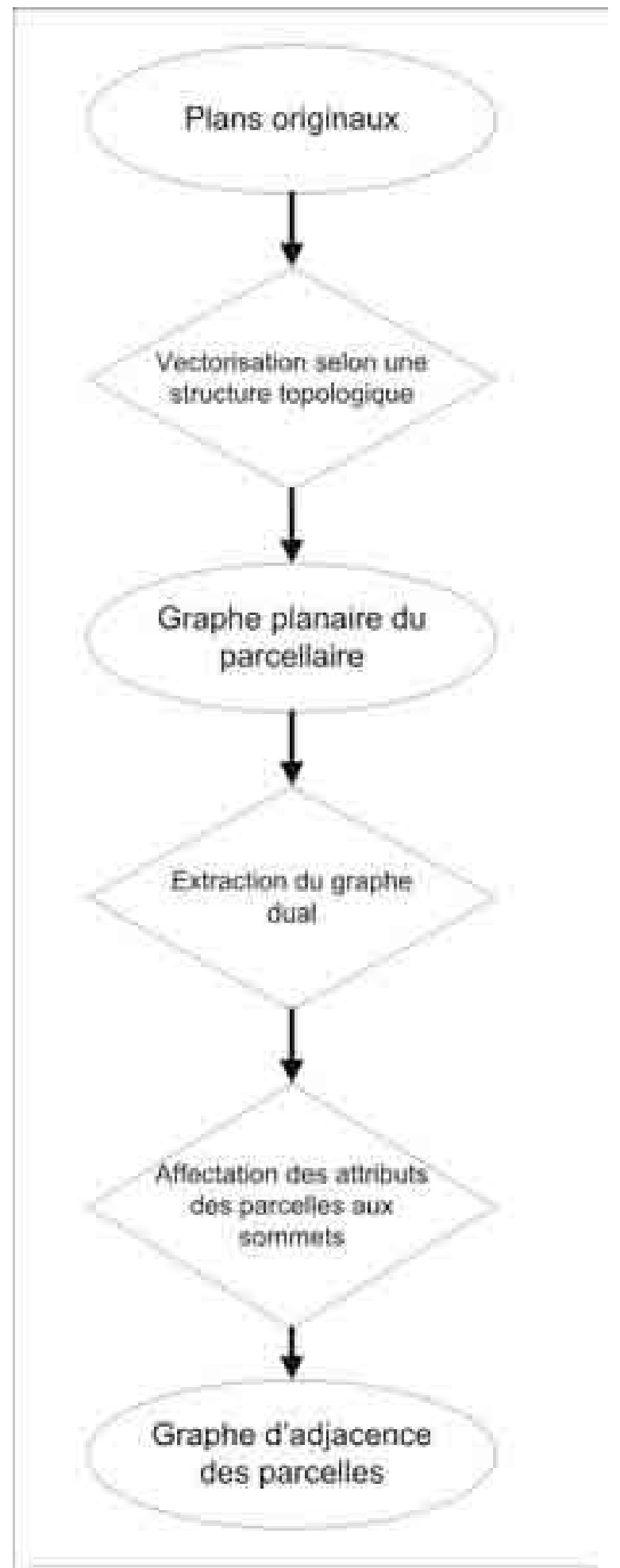

\section{Légende}

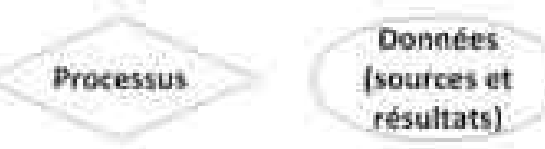

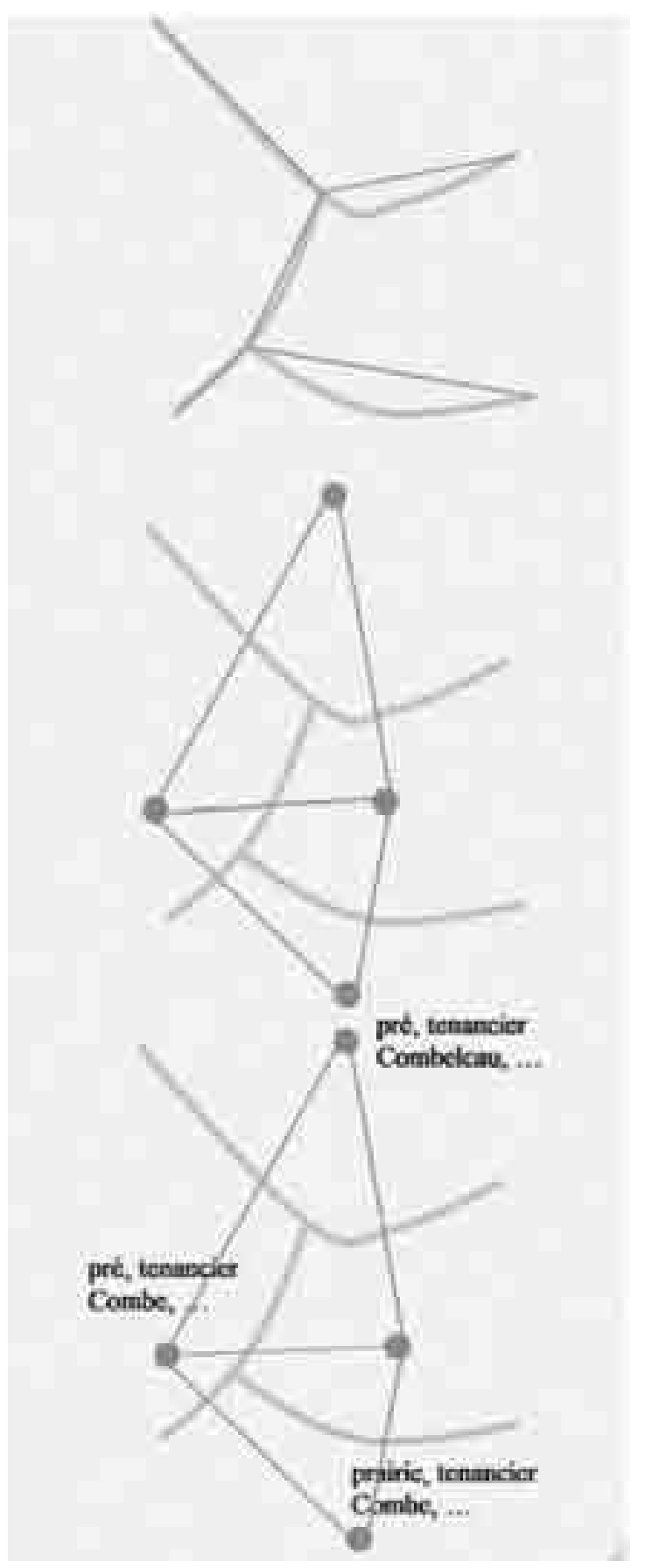

Figure 2 (gauche) : Passage des plans originaux au graphe d'adjacence des parcelles. (droite) : Construction des différents types de graphes à partir d'un parcellaire (limites en traits gris); en haut le graphe planaire topologique, au milieu le graphe dual, en bas le graphe d'adjacence.

Figure 2 (left): Passage from original plans to adjacency graph of plots of land. (right): construction of the different types of graphs from the plots representation (limits in grey); top, the planar topological graph; middle, the dual graph; bottom, the adjacency graph. 
choisir entre plusieurs possibilités quant à l'intégration - ou non - des chemins et des rivières.

\section{La question des linéaires, chemins et rivières}

Le principal problème qui s'est posé au moment d'intégrer les chemins et les rivières au graphe planaire du parcellaire était de savoir où couper les tronçons de chemins. Qu'est-ce qui fait qu'on arrête un tronçon à un endroit et pas à un autre? Plusieurs choix sont en effet possibles pour découper les chemins et, plus largement, pour la manière dont on peut les intégrer au graphe des parcelles; ces choix vont peser sur le graphe que l'on va obtenir ensuite. Les possibilités qui ont été envisagées sont reportées sur le schéma en figure 3.

On peut concevoir :

- (a) un point par chemin. L’idée de départ était de représenter un chemin comme une parcelle, par un seul point, ce qui semble conforme à la description des chemins dans les compoix et terriers. Par exemple, dans le cas de la commune d'Odars (31, France) qui est la fenêtre sur laquelle porte l'expérimentation, la route royale dans le registre cadastral de 1811 correspond à un élément; partout où la route

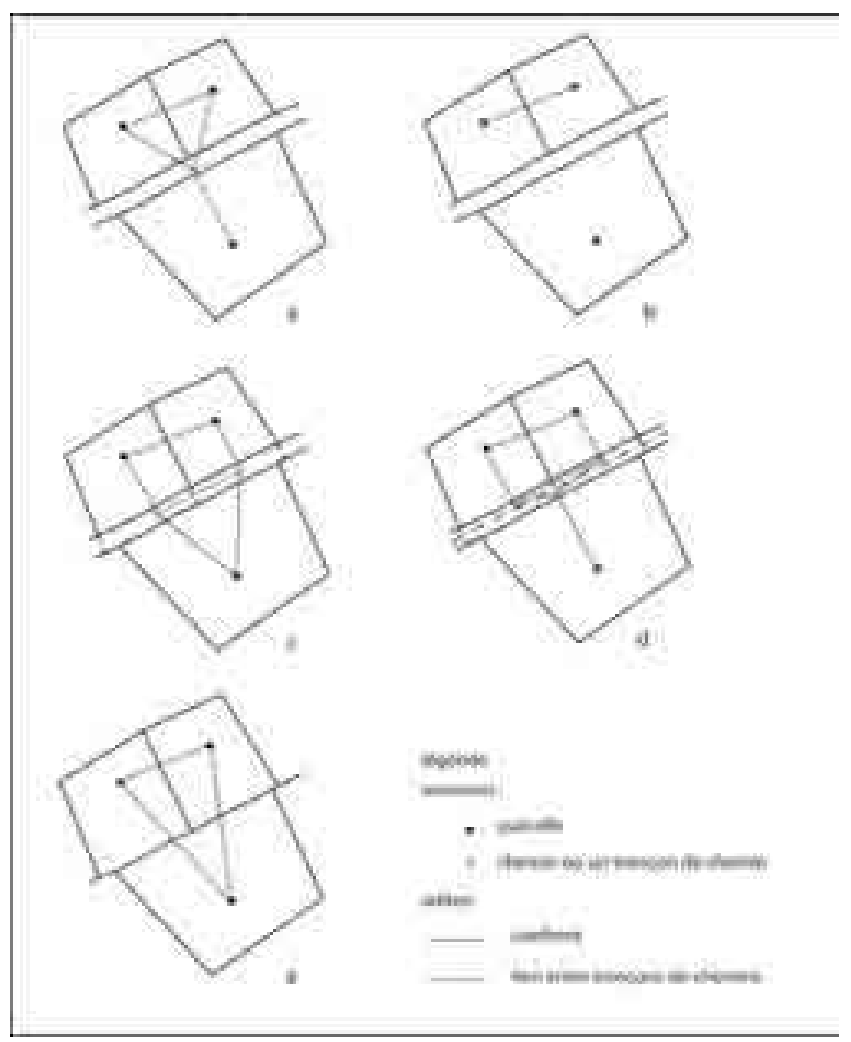

Figure 3 : Possibilités d'intégration des chemins et des rivières au graphe.

Figure 3: Possibilities of integration of roads and rivers into the graph. royale coupe les parcelles, les confronts la nomment. Elle serait représentée par un seul point car elle s'apparente à une entité. Le problème est que la route royale correspond à un cas particulier qui n'apparaît qu'en 1811 ; cette route est parfaitement identifiée sur l'ensemble de son linéaire, mais c'est rarement le cas pour les autres chemins.

Si une grande route peut généralement être résumée à un point sans ambiguïté, les petits chemins sont difficiles à identifier et il est difficile de déterminer les endroits où un tel chemin s'arrête et où un autre démarre; la question se pose en particulier au niveau des carrefours et des pattes d'oies. Faut-il les arrêter à chaque intersection? Couper les chemins à chaque carrefour serait le plus neutre et le plus systématique; les routes deviendraient une chaîne de sommets et d'arêtes. Cependant, on peut se demander si tronçonner les chemins à chaque carrefour ne va pas créer de l'information inutile. L'autre problème du découpage des chemins en tronçons est que, d'un plan à l'autre, de nouvelles intersections risquent d'apparaître, ce qui entraîne de nouvelles difficultés pour les comparaisons de graphes. Néanmoins, ce choix (a) est le plus cohérent du point de vue de la modélisation spatiale de l'information parcellaire puisqu'il offre la meilleure conservation de l'intégrité des objets représentés sur les plans ou décrits dans les registres.

- (b) enlever les chemins et les laisser vides. On obtiendrait plusieurs graphes déconnectés; le graphe total n'est plus connexe. Cette solution pourrait être utile pour comparer les graphes pour lesquels on dispose de plans avec des graphes issus de registres dans lesquels les chemins ne sont pas identifiés. Néanmoins, même si certains chemins ne sont pas identifiés ou nommés, ils peuvent servir comme point de repère. C'est une information qu'il convient de ne pas perdre.

- (c) diviser le chemin en autant de tronçons que de parcelles. La question qui s'est posée est de savoir quel coté du chemin prendre en compte puisque les limites de parcelles ne sont pas forcément face à face, ce qui nous a conduit à envisager la possibilité (d) suivante.

- (d) diviser le chemin en autant de tronçons que de parcelles et en deux dans le sens de la longueur. C'est la solution la plus descriptive, qui se rapproche le plus de ce que décrit le compoix puisque dans les registres, il y a autant de chemins que de confronts de parcelles adjacents aux chemins. Le principal intérêt de cette possibilité est la proximité avec l'image du graphe d'un registre sans plan, mais en contrepartie elle présente plusieurs problèmes. La première difficulté est un changement d'information puisque les sommets représentent des confronts et non plus des chemins. Dans ce cas, on peut se demander pourquoi ne pas représenter tous les confronts par des sommets. Les sommets seraient repor- 
tés sur les pourtours de la parcelle et non au centre de celleci. Ceci n'est pas envisageable en raison de certaines parcelles qui ont des formes en escaliers ou très irrégulières (il serait difficile d'attribuer le même nombre de confronts aux parcelles que celui assigné dans les registres). De plus, c'est la parcelle qui porte l'information et qui est l'objet d'étude premier. Il résulte donc qu'il faut conserver un point par parcelle. Le second problème identifié est que cette solution complique le graphe de manière excessive. Enfin, il apparaitt qu'à partir du registre, on ne connaît pas le voisinage des tronçons de chemins.

- (e) Supprimer les chemins et les intégrer aux parcelles. Les sommets du graphe dual ne représentent alors que les parcelles qui sont reliées directement. Le chemin n'apparaît plus comme un élément, mais comme un attribut, dans la matrice d'adjacence, de l'arête qu'il intersecte. L'information «relation de type chemin » est sur l'arête. L'inconvénient de cette solution est qu'elle demande de modifier le graphe planaire du parcellaire en amont de la construction du graphe dual, en effaçant les chemins et en les intégrant aux parcelles. L'intérêt de cette solution est de conserver l'information des chemins sans compliquer le graphe. Le graphe reste celui des parcelles. De plus, il reste connexe. Cette dernière solution apparaît comme la plus simple, avec le moins de points.

Comme il paraissait difficile de trancher a priori pour la solution la plus pertinente et utile, deux possibilités ont été envisagées après discussions et plusieurs tests; nous avons choisi de tester les solutions (e) où les chemins ne sont pas pris en compte et (a) dans laquelle un chemin est représenté par un point. Suite à ces tests, c'est finalement la solution (e) qui a été retenue.

\section{Procédure technique de construction du graphe dual à partir du plan parcellaire}

Le passage du plan parcellaire au graphe dual s'effectue selon les étapes suivantes à partir d'un fichier de forme contenant les polygones du parcellaire dans le SIG (ArcInfo) :

- Création d'une couverture ${ }^{4}$ de points et d'arcs à partir de la couche de polygones. Ces points sont les sommets du graphe et les arcs les segments qui constituent le contour du polygone. Les sommets sont calculés comme point label, c'est-à-dire un point interne aléatoire au polygone.

- Association des informations de la table attributaire des polygones à chaque point label.

- Vérification de la matrice d'adjacence. La table attributaire de la couche " arc " contient pour chaque arc le polygone de droite et le polygone de gauche. Elle permet

4. Format topologique de données ArcInfo (Pirot 2004 : 422). donc de faire la liste de polygones voisins : si deux polygones ont un arc en commun, c'est qu'ils sont adjacents. Puisque la limite entre deux parcelles correspond parfois à plusieurs arcs, il faut supprimer les arcs redondants. Une fois ces derniers supprimés, on obtient la matrice d'adjacence.

- À partir de cette matrice de la couche des arcs et de la table des sommets, on construit la couche des arêtes du graphe ${ }^{5}$.

\section{RÉsultats}

Après un premier test sur un territoire de Touraine (Le Goffic, 2008), la commune d'Odars (31, France), située à proximité de Toulouse (figure 4), a été retenue comme terrain d'étude en raison de la documentation disponible, à savoir trois compoix ne présentant que des registres datés de $1476^{6}, 1551^{7}$ et $1598^{8}$, un terrier de $1759^{9}$ comportant un registre et un plan, et le cadastre dit «napoléonien » de 1811.

Dans un premier temps, toutes les données contenues dans les compoix ont été dépouillées puis enregistrées dans une base de données qui enregistre les informations spatiales (base de données TERCOMP, cf. ci-dessus). Dans un second temps, les plans ont été traités dans un SIG. Après la numérisation des plans de 1811 et 1759 , leur vectorisation selon une structure topologique a permis d'obtenir les graphes planaires des parcellaires, à partir desquels ont été extraits les graphes duals à l'aide des matrices d'adjacences selon la procédure expliquée ci-dessus. À l'issue de cette étape, deux graphes ont été générés, à savoir celui du plan parcellaire cadastral ancien de 1811 et celui du plan terrier de 1759, en éliminant les chemins et les cours d'eau ou en les prenant en compte (5 et 6). Dans ce dernier, 276 parcelles sont décrites dans le registre du terrier sur les 417 parcelles que comporte le plan.

À ce stade, nous disposons donc de deux graphes correspondant aux états du parcellaire de 1759 et 1811. À partir de ces états connus, deux traitements ont été envisagés : le premier est un test d'appariement de l'état le plus ancien sur le plan parcellaire napoléonien réalisé à l'aveugle, pour tester ce que l'on pourrait obtenir à partir d'un registre complet qui décrirait toutes les parcelles. La seconde piste envisagée

5. Cette opération a été réalisée avec l'outil " création de flux » téléchargeable sur le site http://support.esrifrance.fr/

6. AD31,A MTI I483-1.

7. AD31,2E 4356.

8. AD31,A MTI I487.

9. AD31, AMT II 493. 
Figure 4 : Carte de localisation de la commune d'Odars (31, France).

Figure 4: Map of localization of the district of Odars (31, France).

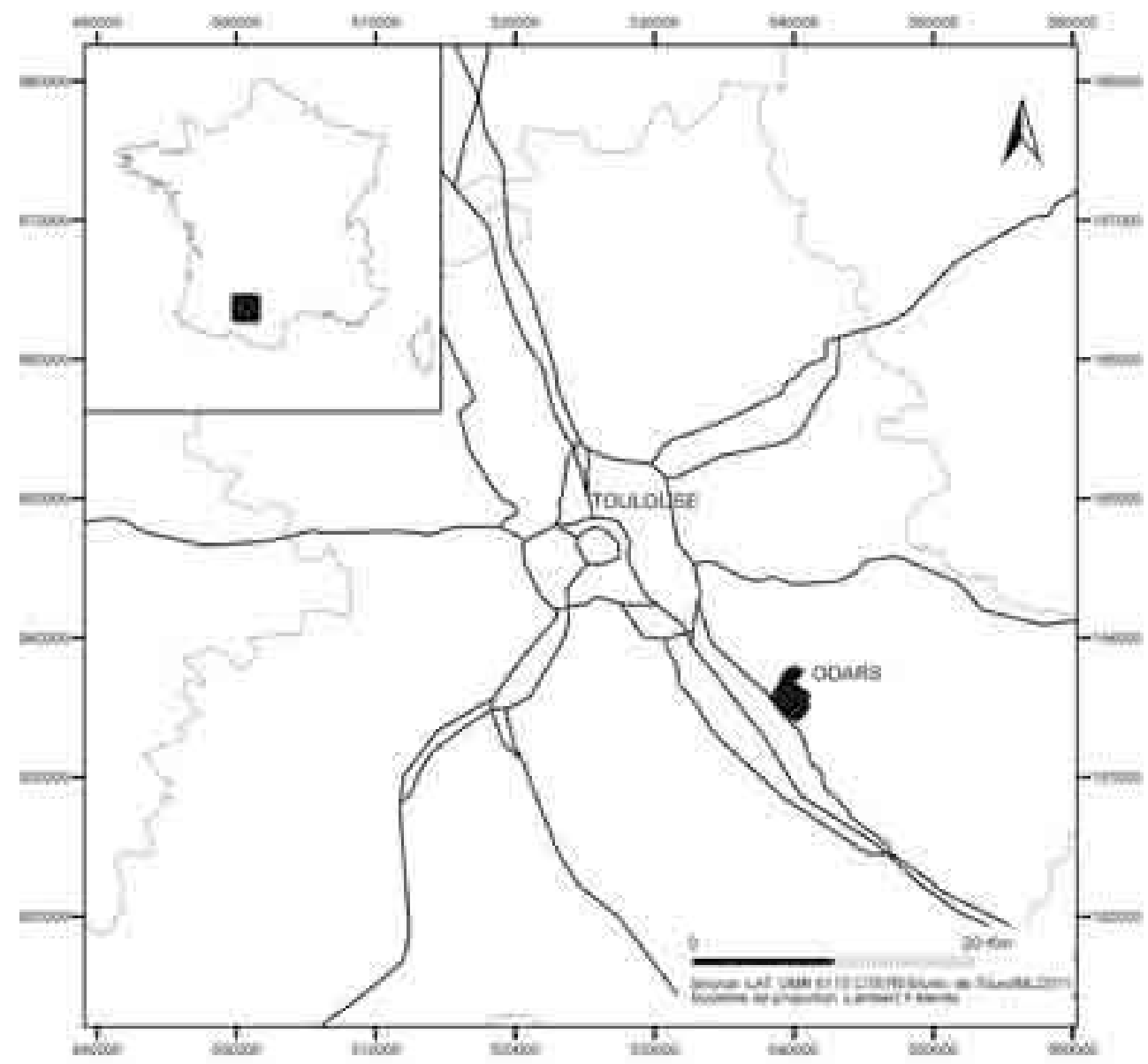

est la comparaison de ces deux graphes soit selon des critères géométriques, soit selon leurs attributs.

Nous avons testé la construction du graphe d'adjacence du plan le plus ancien (1759) à partir de la matrice d'adjacence du plan parcellaire, d'abord sans contraintes de coordonnées ni de projection cartographique, sous un logiciel de manipulation de graphes ${ }^{10}$ avec un algorithme de forces et ressorts (Figure 7). Les algorithmes de forces et ressorts sont des algorithmes de placement pour lesquels les graphes sont présentés comme des systèmes physiques où les arêtes sont des forces d'attractions entre les deux sommets extrémités de l'arête (ressorts). Le système évolue librement vers un placement qui équilibre les forces et constitue souvent une visualisation intelligible de la structure. L'étape suivante a consisté à apparier ce graphe d'adjacence reconstitué au plan parcellaire de 1811 à partir des données du registre. En fait, on a réalisé une simulation permettant d'évaluer les résultats potentiels si l'on arrivait à reconstituer, à partir d'un registre, un graphe sur tout le territoire couvert. L'appariement a été

10. Nous avons utilisé le logiciel Yed Graph Editor, [http://www.yworks. $\mathrm{com} / \mathrm{s}$. réalisé grâce à une procédure d'ajustement spatial, à partir de points d'amer aussi appelés TIC ${ }^{11}$. Différents tests ont été conduits( Figure 8).

Ces tests de construction à partir de la matrice d'adjacence du graphe et de son calage mettent en évidence plusieurs points :

- les éléments linéaires constituent souvent des confronts invariants qui sont importants pour l'appariement des graphes; il est donc essentiel de les prendre en compte dès la construction des graphes, que ce soit à partir des registres ou lors de la vectorisation des plans;

- les toponymes des terroirs sont mobiles; ils se déplacent au fil du temps, ce qui a déjà été mis en évidence par ailleurs (Vue 1997; Vue 2002; Zadora-Rio 2001), mais pose ici la question de leur utilisation comme invariant;

11. Les TIC sont des points de contrôle correspondant à des localisations connues géographiquement. Les TIC permettent d'enregistrer tous les objets géographiques dans un même système de coordonnées. Ils permettent de transformer les " coordonnées table " dans un système de coordonnées, de changer le système de projection. 


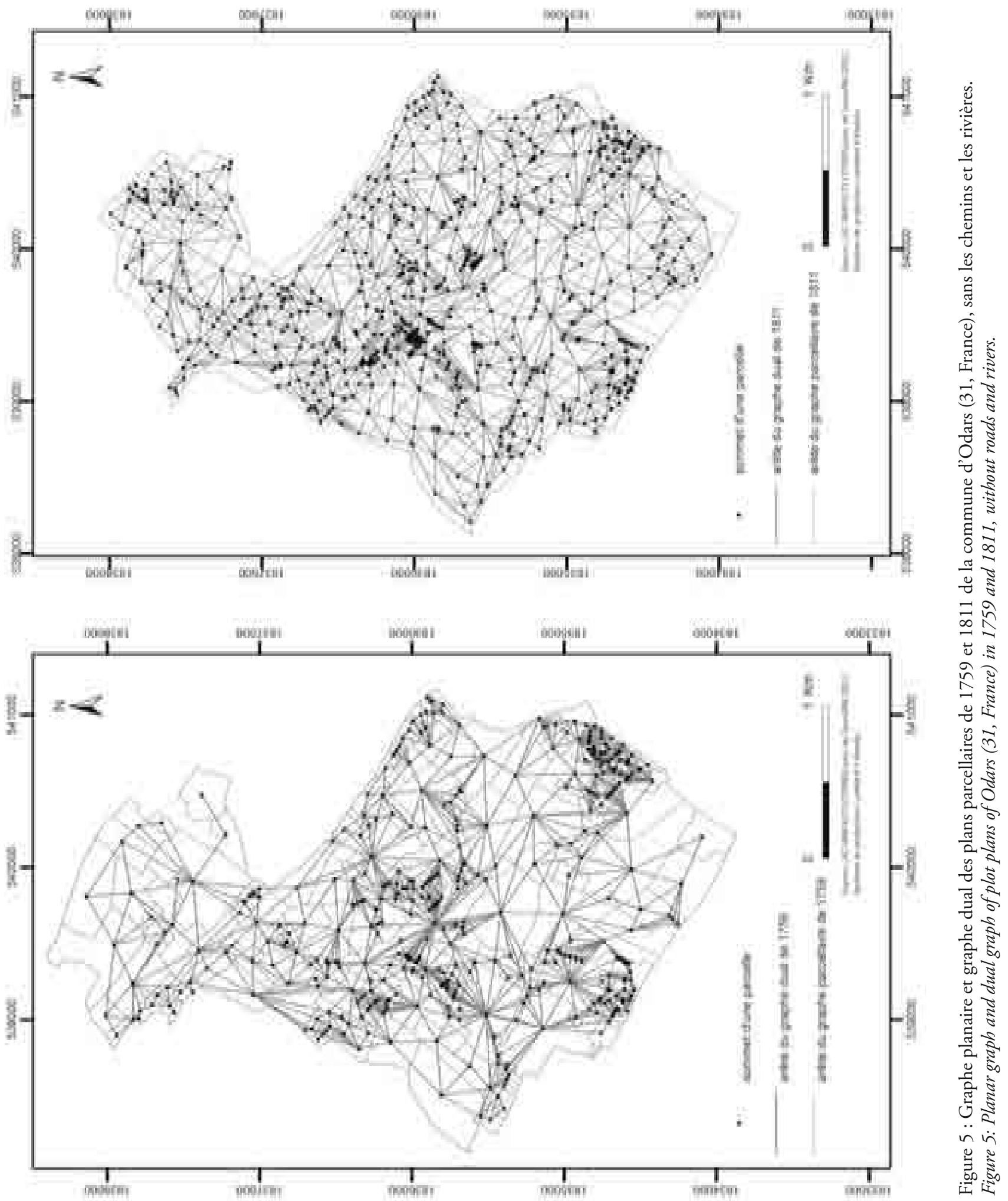




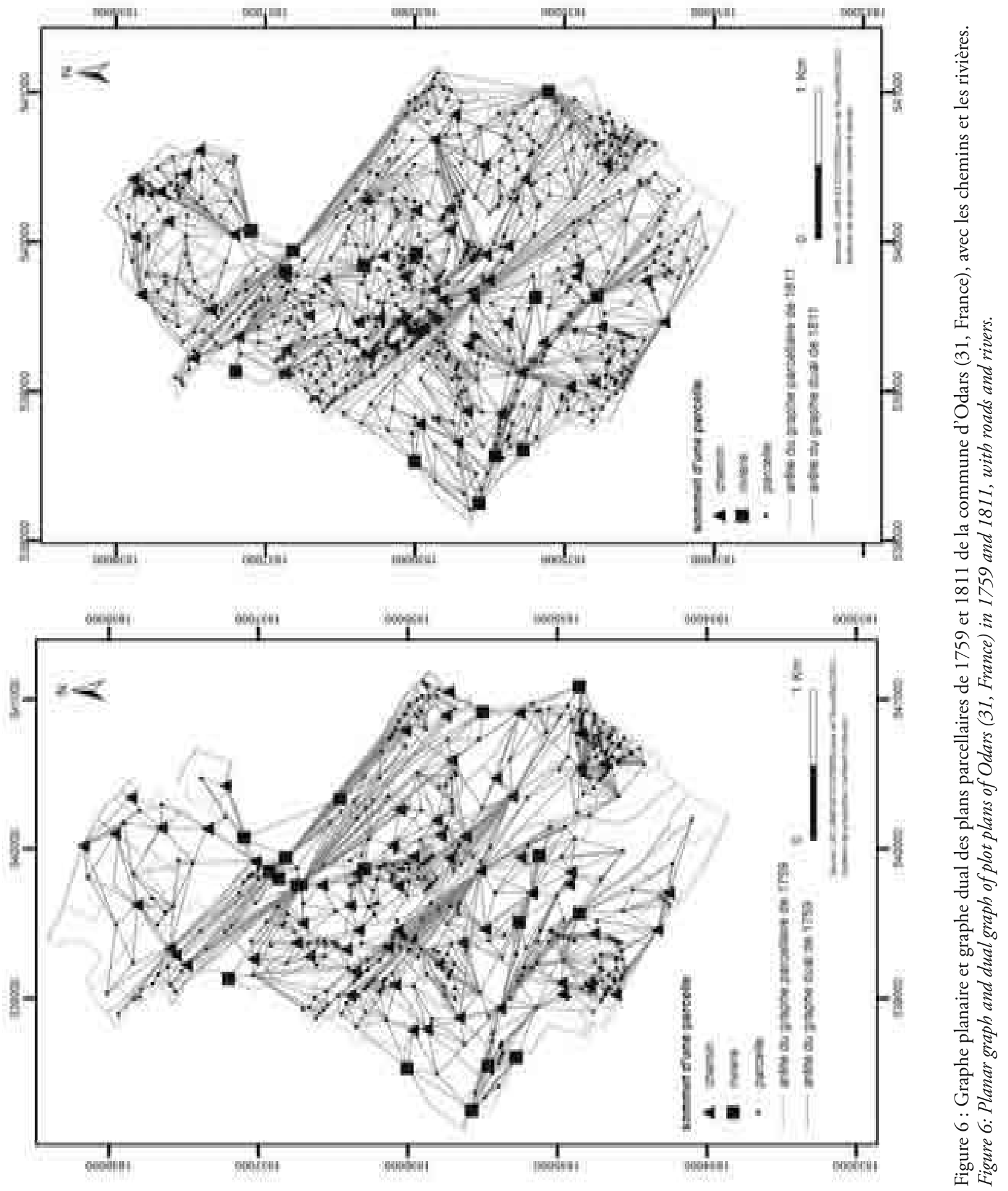



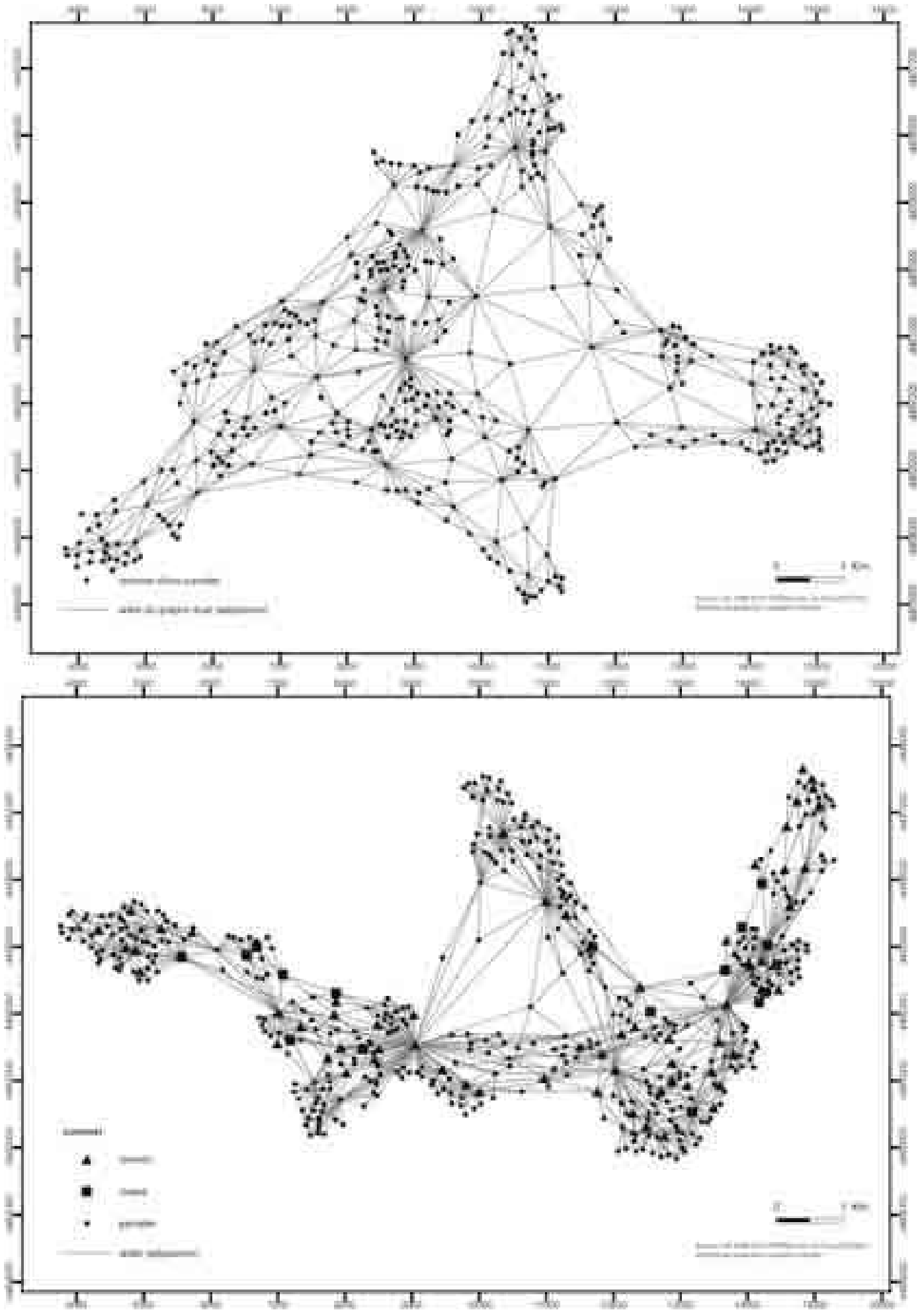

Figure 7 : Graphes duals issus de la matrice d'adjacence du plan parcellaire de 1759 de la commune d'Odars (31, France) après un algorithme de force et ressort sous un logiciel de graphe (en haut, uniquement les parcelles; en bas, avec les chemins et les rivières).

Figure 7: Dual graphs from the adjacency matrix of the plot plan of Odars (31, France) in 1759 with a spring algorithm (at the top, only the plots of lands; below, with the roads and rivers). 


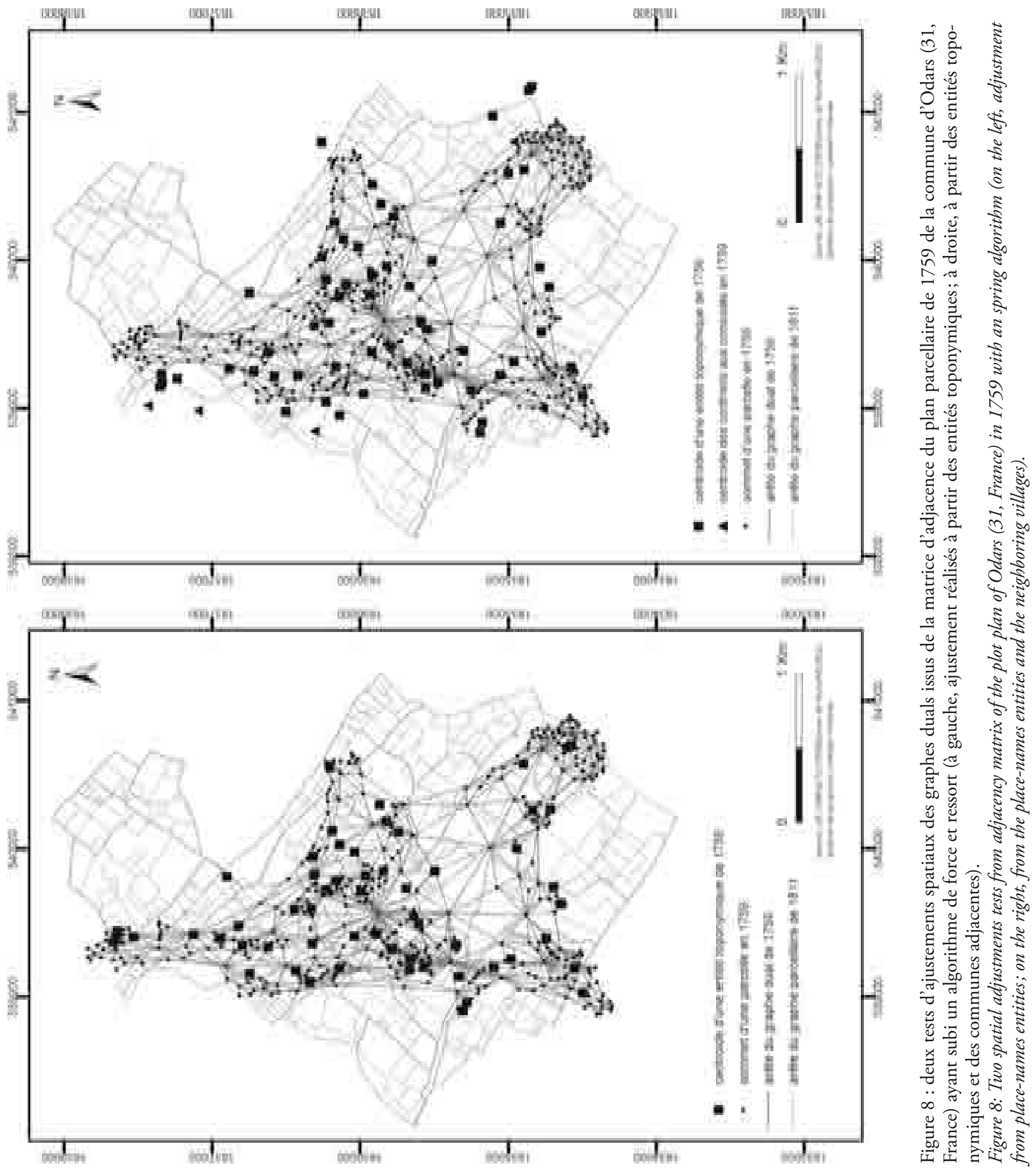




\begin{tabular}{|c|c|c|c|}
\hline Description & Pointsd 'amer & $\begin{array}{l}\text { Nombred e } \\
\text { points }\end{array}$ & Erreur RMS \\
\hline $\begin{array}{l}\text { Test } 1 \text { : Graphe dual sans les éléments } \\
\text { linéaires (chemins et rivières) }\end{array}$ & - centroïdes des entités toponymiques & 20 & 339,78 \\
\hline $\begin{array}{l}\text { Test } 2 \text { : Graphe dual sans les éléments } \\
\text { linéaires (chemins et rivières) }\end{array}$ & $\begin{array}{l}\text { - centroïdes des entités toponymiques } \\
\text { - centrö̈des des parcelles confrontant aux communes } \\
\text { adjacentes }\end{array}$ & 25 & 383,54 \\
\hline $\begin{array}{l}\text { Test } 3 \text { : Graphe dual avec les éléments } \\
\text { linéaires (chemins et rivières) }\end{array}$ & $\begin{array}{l}\text { - centrö̈des des entités toponymiques } \\
\text { - centroïdes des parcelles confrontant aux communes } \\
\text { adjacentes } \\
\text { - centrö̈des des chemins } \\
\text { - centrö̈des des rivières }\end{array}$ & 34 & 884,63 \\
\hline $\begin{array}{l}\text { Test } 4 \text { : Graphe dual avec les éléments } \\
\text { linéaires (chemins et rivières) }\end{array}$ & $\begin{array}{l}\text { - centroïdes des entités toponymiques } \\
\text { - centroïdes des parcelles confrontant aux communes } \\
\text { adjacentes } \\
\text { - centroïdes des chemins } \\
\text { - centroïdes des rivières } \\
\text { En enlevant les points trop distants entre } 1759 \text { et } 1811\end{array}$ & $\begin{array}{l}\text { Traitementen } \\
\text { cours }\end{array}$ & Traitement en cours \\
\hline
\end{tabular}

Tableau 1 : Tableau récapitulatif des tests de calage du graphe dual du parcellaire de 1759 reconstitué d'après la matrice d'adjacence sur le plan parcellaire cadastral de 1811.

*Erreur RMS (ou RMS error, "Root Mean Square Error ", soit la valeur quadratique moyenne, c'est-à-dire la racine carrée de la valeur moyenne des carrés des résidus) : Indicateur de la mesure de la qualité des données. Il représente la différence entre les points de calage appelés TIC originaux et la localisation géographique des nouveaux points de calage calculés par un processus de transformation.

Table 1 : Summary table of adjustements tests of dual graph (1759) from adjacency matrix on the plot plan of 1811.

- l'utilisation des algorithmes de forces et ressorts dans la construction des graphes à partir des registres reste à ce jour à préciser.

Évidemment, les graphes obtenus ne permettent pas de répondre aux questions posées à partir des seuls registres, mais ils permettent d'identifier tous les cas de figure qui existent et de mettre en évidence les problèmes posés par la reconstitution de graphes et l'appariement d'états successifs.

\section{Discussion - Conclusion}

Les résultats obtenus à partir des plans peuvent être mis en perspective avec ceux obtenus à partir des sources écrites qui offrent une information lacunaire, à partir de laquelle on obtient une matrice la plus complète possible. Si les procédures de calage et d'ajustement restent à tester et à calibrer, les graphes obtenus permettent d'ores et déjà d'envisager de travailler sur les comparaisons d'un état à un autre.

Les possibilités de comparaison de ces graphes pourront être testées à l'aide du SIG selon deux approches. Dans un premier temps, les transformations spatiales seront étudiées. Il s'agit de mettre en évidence, à partir des graphes, les recompositions parcellaires à l'œuvre, c'est-à-dire recon- naître, dans le passage d'un graphe à l'autre, les opérateurs spatio-temporels de changements pertinents dans le cas des parcellaires (créations, stabilités, disparitions, dilatations, contractions, déformations, fissions, fusions). Ceci pourra se faire dans un premier temps en se restreignant à la comparaison des voisinages des points d'amer. Dans un second temps, on peut envisager d'exploiter les attributs des sommets décrivant les parcelles (usage du sol, surface, propriétaire) afin de mesurer les continuités/discontinuités. Il sera aussi possible de comparer les indices de mesure de structure classiquement utilisés sur les graphes (distribution de degrés, de centralité...), indicateurs de la dynamique.

\section{Remerciements}

Ils vont à Ludovic Granjon, Assistant ingénieur à l'université de Bourgogne, MSH de Dijon, pour sa contribution à la manipulation des matrices d'adjacence. 


\section{Bibliographie}

AткіN, R.H., 1977 - Combinatorial connectivities in social systems : an application of simplicial complex structures to the study of large organizations, Basel, Birkhäuser.

BABson, E. et al., 2006 - Homotopy theory of graphs, Journal of Algebraic Combinatorics, 24, p. 31-44.

Barabási, A.-L. et Albert, R., 1999 - Emergence of scaling in random networks, Science, 286, p. 509-512.

Berge, C., 1958 - Théorie des graphes et ces applications, Dunod, Paris.

Berge, C., 1970 - Graphes et Hypergraphes, Dunod, Paris.

Biget, J.-L., Hervé, J.-C. et ThéBert, Y., 1989 - Les cadastres anciens des villes et leur traitement par l'informatique, École française de Rome, Rome.

Boulet, R., Fieux, E., Jouve, B., 2010 - Simplicial simple-homotopy of flag complexes in terms of graphs, European Journal of Combinatorics 31, p. 161-176.

Brunel, G., Guyotjeannin, O. et Moriceau, J.-M., 2002 Terriers et plans-terriers du xiiie au xviiie siècle. Actes $d u$ Colloque de Paris (23-25 septembre 1998), Caen/Paris, Association d'Histoire des Sociétés Rurales/École Nationale des Chartes.

Cantwell, M.D. et Forman, R.T.T., 1993 - Landscape graphs : Ecological modeling with graph theory to detect configurations common to diverse landscapes, Landscape Ecology, 8(4), p. 239-255.

Chen, B., Yau, S.-Tung et Yeh, Y.-nan, 2001 - Graph homotopy and Graham homotopy, Discrete Mathematics, 241(1-3), p. 153-170.

Claveirole, A. et Pélaquier, É., 2001 - Le compoix et ses usages. Actes du Colloque de Nîmes - 26 et 27 novembre 1999, Publications de l'Université Paul Valéry-Montpellier 3, Montpellier.

Conesa, M., 2007 - Capbreu et paysage. Remarques sur l'utilisation d'une source notariale dans l'étude des paysages des Pyrénées de l'est, (Cerdagne, $\mathrm{XVI}^{\mathrm{e}}$-XviII ${ }^{\mathrm{e}}$ siècle), LIAME, Bulletin du centre d'histoire moderne et contemporaine de la Méditerranée et de ses périphéries, 14, p. 97-124.

Dale, M.R.T. et Fortin, M.J., 2010 - From Graphs to Spatial Graphs, Annual Review of Ecology, Evolution, and Systematics, 41, p. 21-38.

Dochterman, A., 2009 - Homotopy groups of hom complexes of graphs, Journal of Combinatorial Theory, 116(1) , p. 180-194.
Le Goffic, B., 2008 - Modélisation et analyse du parcellaire à l'aide la théorie de graphes, Master Archéomatique, Université François-Rabelais de Tours, France.

Leturce, S., 2007 - Un village, la terre et ses hommes. Toury en Beauce (xiie-xviie siècle), $\mathrm{P}$ aris, C THS.

Montpied, G. et Rouault, J., 1982 - Reconstitution et cartographie d'un parcellaire à partir de cadastres textuels $\mathrm{du} \mathrm{Xv}^{\mathrm{e}}$ siècle, Le médiéviste et l'ordinateur, 8, p. 11-13.

Montpied, G. et Rouault, J., 1989 - Du texte au graphe : établissement d'une carte du parcellaire à partir des données de deux cadastres de la fin du Moyen Âge. In J.-L. Biget, J.-C. Hervé, et Y. ThéBert (éd.). Les cadastres anciens des villes et leur traitement par l'informatique, École française de Rome, Rome, p. 359-380.

Poirier, N., 2010 - Un espace rural à la loupe. Paysage, peuplement et territoires en Berry de la préhistoire à nos jours, Presses universitaires François-Rabelais, Tours.

Pirot, F., 2004 - Glossaire. Histoire et mesure, 3-4, vol. XIX, p. 422 et ss. (Dossier "Système d'information géographique, archéologie et histoire ").

Truel, Y., 2007 - Représentation fiscale au xviie siècle : le HautSégala quercynois. Le compoix de Saint-Cyrgues (1636). Mémoire de Master 2, Université de Paris I Panthéon-Sorbonne, France.

Urban, D.L. et KeItT, T.H., 2001 - Landscape connectivity : a graph-theoretic perspective, Ecology, 82, p. 1205-1218.

Vue, B., 1997 - Microtoponymie et archéologie des paysages à Neuilly-L'évêque (52) du 13e au 20e siècle : comportement des microtoponymes au fil des siècles: le nom, l'espace et l'homme qui le nomme, Thèse de doctorat de linguistique, Université de Nancy 2, France.

VuE, B., 2002 - Microtoponymie et archéologie des paysages : huit siècles de comparaison en Pays de Langres, dans Dans La vigne et les vergers, actes du 10e colloque d'onomastique, Université de Reims (30 septembre-2 octobre 1999). Publications de l'université de Reims, Reims, p. 161-181.

Watts, D.J. et Strogatz, S.H., 1998 - Collective dynamics of «small-world» networks. Nature, 393, p. 440-442.

Zadora-Rio, E., 2001 - Archéologie et toponymie : le divorce, Les petits cahiers d'Anatole, 8. Available at: [http://www.univ-tours. frllat/pdflF2_8.html]. 